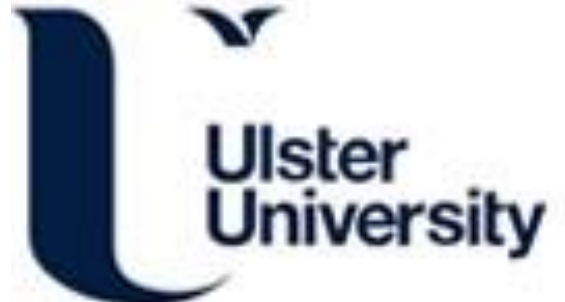

\section{From Paper to Play - Design and Validation of a Smartphone Based Cognitive Fatigue Assessment Application}

Price, E., Moore, G., Galway, L., \& Linden, M. (2016). From Paper to Play - Design and Validation of a Smartphone Based Cognitive Fatigue Assessment Application. In Lecture Notes in Computer Science (Vol. 10069, pp. 321-333). Springer. https://doi.org/10.1007/978-3-319-48746-5_33

Link to publication record in Ulster University Research Portal

\author{
Published in: \\ Lecture Notes in Computer Science
}

Publication Status:

Published (in print/issue): 02/11/2016

DOI:

10.1007/978-3-319-48746-5_33

\section{Document Version}

Author Accepted version

\section{General rights}

Copyright for the publications made accessible via Ulster University's Research Portal is retained by the author(s) and / or other copyright owners and it is a condition of accessing these publications that users recognise and abide by the legal requirements associated with these rights.

\section{Take down policy}

The Research Portal is Ulster University's institutional repository that provides access to Ulster's research outputs. Every effort has been made to ensure that content in the Research Portal does not infringe any person's rights, or applicable UK laws. If you discover content in the Research Portal that you believe breaches copyright or violates any law, please contact pure-support@ulster.ac.uk. 


\title{
From Paper to Play - Design and Validation of a Smartphone Based Cognitive Fatigue Assessment Application
}

\author{
Edward Price $^{1}$, George Moore ${ }^{2}$, Leo Galway ${ }^{2}$, and Mark Linden ${ }^{3}$ \\ ${ }^{1}$ Ulster University, School of Computing and Mathematics, United Kingdom \\ price-e@email.ulster.ac.uk \\ ${ }^{2}$ Ulster University, School of Computing and Mathematics, United Kingdom \\ $\{$ g.moore, l.galway\} @ulster.ac.uk \\ ${ }^{3}$ Queens University Belfast, School of Nursing and Midwifery, United Kingdom \\ $\mathrm{m}$. linden@qub.ac.uk
}

\begin{abstract}
This paper investigates the user experience design of a smartphone application for the objective assessment of cognitive fatigue. This is as an alternative to using an established paper questionnaire that offers subjective selfassessment. Taking a multidisciplinary approach, challenges relating to the usability and the efficacy of the smartphone assessment tool were explored. Furthermore, to enable validation of the proposed new approach, challenges relating to how best to deliver the traditionally paper-based questionnaire on a smartphone display, while retaining the validity of the measure it affords, had to be addressed. Results show that the smartphone based cognitive testing methods was comparable to outcomes from the pre validated mobile based Mental Fatigue Scale. Participant feedback showed that the smartphone-based approach offered a more acceptable and engaging user experience, while retaining the ability to accurately measure cognitive fatigue.
\end{abstract}

Keywords. Mobile $\cdot$ questionnaire adaptation $\cdot$ cognitive tests $\cdot$ fatigue evaluation $\cdot$ self-assessment.

\section{$1 \quad$ Introduction}

Fatigue can be defined as "the awareness of a decreased capacity for physical and/or mental activity due to an imbalance in the availability, utilization, and/or restoration of (physiological or psychological) resources needed to perform activity" [1]. Fatigue is often caused by a lack of sleep, stress, or a cognitive deficiency. Cognitive fatigue is a common symptom that may occur after an Acquired Brain Injury (ABI), and can have a very detrimental effect on a person's ability to conduct activities of daily living. Measuring cognitive fatigue is considered problematic due to the absence of biological markers and a lack of standardized tests. This results in an absence of available technology-based solutions [22]. Consequently, episodes of fatigue can potentially be misinterpreted. 
This paper investigates the user experience design of a smartphone application for the objective assessment of cognitive fatigue. This is as an alternative to using an established paper questionnaire that offers subjective self-assessment. Taking a multidisciplinary approach, challenges relating to the usability and the efficacy of the smartphone application assessment approach were explored. It also aimed to validate its use on a smartphone alongside objective methods that would previously have been deployed using large screen-based devices. Furthermore, it anticipates the collaborative use, or eventual replacement, of traditional self-assessment methods with pervasive computing approaches. Self-assessment of fatigue most frequently takes place within a clinical environment, which doesn't necessarily facilitate the convenient and timely self-reporting of fatigue during everyday life. However, tests such as the Psychomotor Vigilance Task and Spatial Span Task can be adapted for mobile devices, which, in turn, could be utilized to provide more effective and accurate fatigue assessment. Results show that the mobile-based approach offered a more acceptable and engaging user experience, while retaining the ability to accurately measure cognitive fatigue.

The remainder of this paper provides an overview of the background and related work in this area. This is followed by discussion of the application design utilized for the research conducted within this paper, which indicates how relevant research has been used to inform the design of the related smartphone application. The results from a preliminary study using the application will be subsequently presented, and finally, concluding remarks and future work will be discussed.

\section{Related work}

\subsection{Subjective Assessment}

Traditional methods of subjective assessment in the area of cognitive fatigue have utilized self-assessment questionnaires as a way of measuring an individual's perceived level of fatigue. There have been a number of specific self-assessment questionnaires designed to target cognitive ability and its relation to fatigue, including the Visual Analogue Scale for Fatigue [18], Fatigue Severity Scale [15] and the Mental Fatigue Scale (MFS) [13]. All of these scales use a visual analogue representation of targeted questions in order to aid a participant in self-evaluation. Moreover, the validity of such scales has been evaluated and shown to be effective in their assessment capabilities [16]. However, as no medical standard for fatigue measurement currently exists, different scales are available depending on whether or not the physical or emotional aspect is being evaluated.

The MFS is the first scale that has been adapted to assess fatigue irrespective of the underlying neurological condition. Similar to the previous scales, this scale takes the form of a self-assessing questionnaire, which consists of 15 questions specifically aimed at covering the main symptoms that occur after a brain injury, including cognitive symptoms, sleep deprivation and duration, and other common effects [11]. Questions do not focus specifically on any particular area so that a wider understanding of fatigue can be garnered. In addition, the scale has been shown to be invariant to age, 
gender and educational profile [2]. Johansson measured the effectiveness of the MFS against a series of neuropsychological tests in order to prove its validity [12]. The tests used were; digit symbol-coding from the The Wechsler Adult Intelligence Scale (WAIS)-III [21], measuring information processing speed; the digit span from the WAIS-III, measuring attention and working memory [21]; verbal fluency test [19] and Trail Making Test (TMT) A and B [17], measuring visual scanning, divided attention and motor speed.

As self-assessment is a key approach to monitoring fatigue, it has great potential to be adapted and deployed onto mobile platforms. Within the research literature, Swendeman et al. [20] carried out a study into the validity of self-reporting via a smartphone. They evaluated behavioral and emotional self-reports daily over a 6-week period. Daily completion rates of surveys were reported to be $50 \%$, with $70 \%$ of participants completing three follow up surveys after the 6-week period. Adherence to the daily assessment was observed to be low, which was attributed to errors in data that subsequently had to be excluded from the evaluation results. It may indicate the monotonous nature of self-assessment for participants, regardless of medium, which subsequently highlights the overall need for a more engaging approach.

\subsection{Objective Assessment}

The WAIS was originally designed to measure intelligence but has since been adopted by neuroscientists in order to evaluate cognitive performance of brain-damaged individuals in terms of processing speed, reaction and memory [2][12].

Van Dongen [6] defined three computerized methods for assessing fatigue after chronic sleep restriction: (1) a mental arithmetic test to assess cognitive throughput; (2) a sustained reaction time test; (3) a digit-symbol substitution task to assess memory. The experiment yielded convergent findings of sleep dose-response effects on all three cognitive performance functions. Specifically, sleep periods limited to four hours and six hours per night progressively eroded the effectiveness of psychomotor vigilance performance, working memory performance and cognitive throughout performance, thus providing evidence for the adverse effects of chronic sleep restriction and fatigue on cognitive function. While many technology-based approaches exist that are capable of assessing fatigue within a clinical environment, it is important to also develop mobile based methods, which would potentially facilitate assessent outside of the clinical environment.

The Psychomotor Vigilance Task (PVT) was originally designed for a static computer-based evaluation, however, it is one of the few tests that has been modified for use on mobile platforms. Work by Kay [14] and Gartenberg [8] investigated how effective short duration mobile tests would be, in conjunction with the usability issues that arise. They concluded that mobile-based variations were just as effective as desktop-based assessment, thus highlighting the adaptability of the underlying cognitive tasks. However, the assessments employed only focused on measuring vigilance as an evaluation of alertness and would have benefited from additional measurements such as working memory and throughput. 
The Spatial Span Task used by Johansson, taken from the WAIS, measures the same cognitive attention and working memory ability as the Digit-Symbol Coding Task defined by Van Dongen as a metric to measure fatigue [6]. The key difference between the two tasks lies in the simplicity of the Spatial Span Task, which proves easier for individuals with to actively engage in. Furthermore, its simplicity further supports development and deployment on a mobile platform.

Johansson [9] compared the ability of a mental fatigue questionnaire with cognitive tests in order to determine if there is a direct correlation in their ability to subjectively and objectively measure fatigue. The neuropsychological tests employed included Digit Symbol-Coding Task, Digit Span Task, Spatial Span Task [19] and Trail Making Tests [17]. Accordingly, these tests were employed to measure processing speed attention, working memory, verbal fluency and reading speed. It was concluded that subjective mental fatigue mainly correlates with objectively measured information processing speed [9].

\section{$3 \quad$ Methodology}

This research employed a multi-disciplinary, iterative approach to systematically inform and evaluate each aspect of the smartphone application. Experts from the field of acquired brain injuries, psychology and interaction design were involved in informing initial design decisions and function of the application, alongside user pilot studies. This permitted the design and development of the smartphone application to be informed by clinical theory and practice, as well as commercial design theory and user opinion. This iterative design process is overviewed in Figure 1.

The designed smartphone application utilized a variation of the MFS for use on a small screen. To ensure impartiality, the original paper design of the questionnaire has been utilized for the mobile variation employed, including the visual design of the questions, the sequencing of questions, and numbering of the scale. Its validity has previously been assessed alongside a series of neuropsychological tests [10]. Consequently, it was chosen for adoption in the smartphone application discussed herein, together with three different cognitive tests: (1) the Spatial Span Task from WAIS; (2) the PVT; (3) Serial Addition/Subtraction Task. Correspondingly, the cognitive tests selected require sufficient cognition to evaluate multiple different areas of affect, including memory, attention, speed of processing and cognitive throughput.

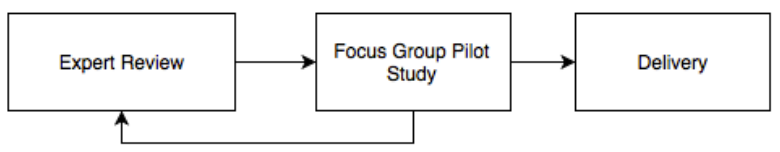

Fig. 1. Iterative Design Process 


\subsection{Expert Review}

The initial phase of the design process was focused on clinical consultation and expert knowledge in order to inform the workflow of the application. Ordering of tests was considered as a crucial step, as retaining user attention while they might be cognitively fatigued is a difficult challenge. Consequently, through clinical expertise, ordering of tasks was based on the perceived level of task difficulty in conjunction with the perceived level of user enjoyment for individual tasks. As such, more difficult tasks were sequenced earlier, whereas more engaging tasks were sequenced later, in order to retain attention and engagement as the tasks become increasingly more stimulating. In addition, providing a 'fun' task after a 'boring' task gives the user an incentive to continue, which may be potentially helpful under fatigued circumstances. Subsequently, the ordering of the task as presented by the application is MFS, Spatial Span Task, PVT and Serial Addition/Subtraction Task. The overall application test takes no more than 10 minutes to complete, with the duration selected to ensure better user engagement as longer time on tasks can lead to a level of degradation in participation and a lower level of perceived effort. Expert review was carried on alongside the next stage of focus group testing.

\subsection{Focus Group Pilot Study}

Next, the initial version of the proposed smartphone application was delivered to a small group of participants $(n=5)$, recruited from the Ulster University, in order to investigate how information design and visual design choices made during expert review might affect the usability of the application. This was also an important stage as it permitted evaluation of the difficulty of each of the cognitive tests; if they were too complex they would be difficult for users to follow, however, by contrast, if they were too simplistic, they might not produce enough variability in the results obtained for conducting an effective degree of data analysis. For increased usability the application included an instructional screen before each of the cognitive tests. This was included to help users understand the nature and goal of the tests and to reduce the number of mistakes that were recorded by users during the pilot study.

Designing a smartphone application approach to a paper-based questionnaire produces many design and usability challenges. Careful consideration is needed though each stage of the iterative design process in order to take into account both expert opinion as well as user feedback. The Mental Fatigue Scale has 15 questions, each with 7 possible response options. On a paper-based format, it is easy to present all of this information on a page, however, on testing with a small-screen based mobile device, the original format of the questionnaire proved to be too complex and a modified approach was required. Consequently, each question and response option was given its own screen; this permitted the user to focus on just one question before having to think or be overloaded with new information. In addition, it also removed the need to make use of a long scrolling screen for questions, which would potentially lead to misunderstanding of the current question. Figure 2 illustrates one of the question screens for the modified questionnaire; each question is presented and the user re- 
sponse must be obtained before moving on to the presentation of the next question. In order to improve accessibility, feedback from the pilot study showed that response options should have an indicator to highlight user selections, which was added to the final version of the smartphone application. Through the pilot study, feedback and clinical expertise, the MFS component of the application was modified to more closely follow the design and functionality of Apple's ResearchKit [23,24] survey delivery design. This included use of simplistic design, visual layout of questions and answer format.

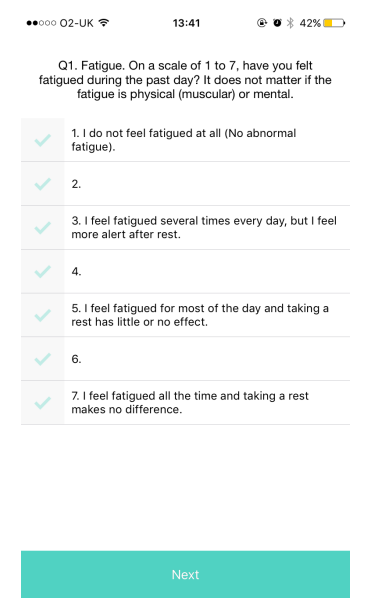

Fig. 2. Example Question Screen

Second stage of the overall application was the delivery of the Spatial Span Task. This was initially designed with a $5 \times 5$ grid layout, however, feedback from the pilot study indicated the proposed layout was too complex for the size of the screen, which resulted in making each square more difficult to select on touch-screen devices. In addition, iOS Human Interface Guidelines recommend any touch-screen element that is to be pressed should be $44 \times 44$ pixels, so the grid was subsequently reduced to $4 \times 4$ squares to accommodate this, as illustrated in Figure 3a. The Spatial Span Task employs a limit of 90 seconds for the user to complete as many sequences as possible, so a countdown timer was added to the top-right of the screen in order to inform the user of the remaining time during the test. Due to the possible, varied lengths of the current sequence to be reproduced by the user, a progress bar was also included at the bottom of the screen in order to provide additional visual feedback to the user. Similar to the modified design of the MFS, this section of the application also followed the design principles used in Apple's ResearchKit [23,24] Spatial Memory Task with only a few small adjustments in an effort to simplify the overall task for users. As a result, the grid design used is somewhat more simplistic than that used by Apple in attempts to reduce the on-screen complexity and potential for distraction. The addition of a sequence progress bar also provides real-time feedback to users for improved usability.

The PVT requires participants to react, as quickly as possible, 15 times to an on screen prompt. Initial design reviews showed that having a just a single prompt appear 
in the same position on screen allowed participants to preempt when to tap the stimulus. As preempting a response breaks the reactive nature of the test, and would not give an accurate indication of a user's fatigue levels, it was decided that a varying stimuli was needed. This was realized by splitting the screen in half and randomizing which side produced a stimulus in order to eradicate the preemptive responses in the task, as shown in Figure 3b. Early or preemptive reactions resulted in a red screen stating the error. Correct reactions display the reaction time on screen, by way of providing feedback of task completion to the participant. This task differs from the reaction time task provided by Apple's ResearchKit in two main ways. Firstly, our approach has a varying stimulus in an attempt to minimize preemptive responses. Also, the input modality is different, as in the ResearchKit version the user is required to shake the device in response to a stimulus. However, in testing this approach it was found that it resulted in a higher rate of errors than a finger press, and so for the final application the finger press was chosen to be included.

The final test delivered by the smartphone application was the Serial Addition/Subtraction Task. Through the iterative user centered development approach employed, feedback from user experience expert highlighted the layout of the keyboard, as illustrated in Figure 3c as a potential concern. During the pilot study, some participants viewed the keypad as being the incorrect orientation for a calculator-based interaction as they are traditionally laid out with the number sequence 1-2-3 on the bottom row. However, most participants considered the number layout shown in Figure $3 \mathrm{c}$ as a more familiar keypad, similar to that used for smartphone calling applications, which are used more regularly than keyboard number pads with a calculator layout. Consequently, it was decided that layout would be used instead of the reverse calculator button layout.

All acquired data collected during assessments is stored internally by the smartphone application once a test is completed and is subsequently transmitted for external storage on a secure server that hosts a back-end database. Storing the acquired data on the device allows for instances when there is little or no network connectivity available, thereby preventing potential loss of test responses. In such cases, when network connectivity is restored the test data is transmitted to the back-end server. This is good practice for alleviating frustration on the user's part, which could be caused by any lost data and having to retake a test. 


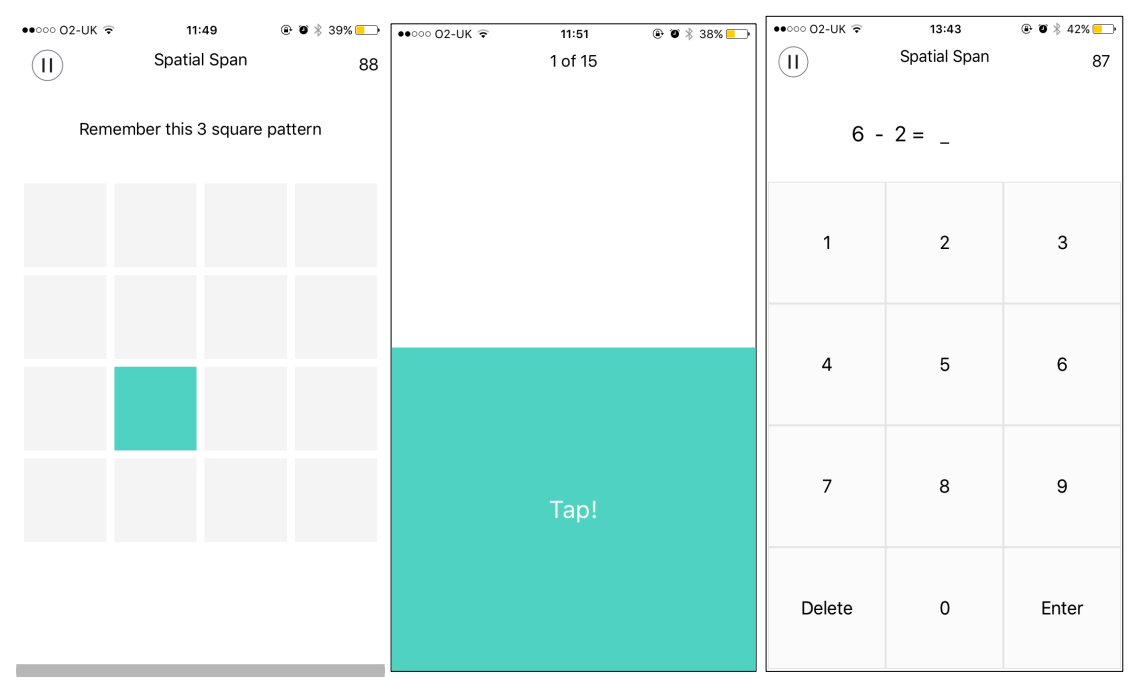

Fig. 3a. Spatial Span Task Fig. 3b. PVT, Figure 3c. Serial Addition/Subtraction

\subsection{Notifications to Promote Participation}

Task prompting has been widely employed as an effective compensatory method for memory impairments [4][5]. To help increase participation with daily testing using the smartphone application, notifications are periodically issued to the smartphone in order to prompt the user to carry out the sequence of tests. If the tests have already been performed on a given day, then no prompt will be shown. Prompting also removes the pressure from the user of having to remember to participate in the test each day.

\section{$4 \quad$ Results \& Discussion}

\subsection{Design Results}

Through the use of a multidisciplinary iterative approach, design considerations were able to be addressed before deployment in a main study. Expert review and a focus group pilot study allowed the design of the smartphone application to be incrementally adapted in response to concerns and feedback. Each of the three different cognitive tests received significant design revision after scrutiny from clinical expertise and user feedback. The Spatial Span Tasks grid size was reduced to $4 \times 4$ in response to user feedback and results which showed that a $5 \times 5$ grid was too complex to process and didn't give a wide enough variability in results. Though user testing in the pilot study it was shown that the initial design of the PVT task allowed participants to preempt the prompt and so this was addressed by varying the location of the stimuli on screen. This increased the reactive nature of the test which give a better measurement of a participants speed of processing. Finally, the keyboard layout of the Serial Addi- 
tion/Subtraction Task was addressed through expert review as it was initially thought that the layout might been seen by participants as upside down for a calculator based task. User feedback from the pilot study indicated that this wasn't the case and that the layout provided was familiar due to its being the same orientation that is used for smartphone calling applications.

\subsection{Study Results}

Immediately after the study had been completed users were required to complete a System Usability Scale questionnaire [3]. This is one of the most widely used usability questionnaires and was chosen in this case as it is technology independent, being able to assess the usability of a wide range of software types, including smartphone applications. Additionally, participants were also asked to freely write what they found the most and least enjoyable aspects of the smartphone application. Participants $(n=21)$ were recruited to participate over a two-week period. The mean age of participants recruited was 22 years $(\mathrm{SD}=4)$. Consequently, 81 individual testing instances were recorded. Significantly, due to the independent nature of each set of data resulting from a test run by a participant, all resulting statistical analysis was able to consider each set of results as an independent data point.

After the study was carried out, $81 \%$ of participants took part in the follow up usability questionnaires. From the System Usability Scale, the overall application received and average score of 74, indicating an above average rating for its ease of use. Through the open comments it was seen that the biggest drawback of the application was the MFS and its perceived tedious nature. Results from this evaluation indicated that the primary reason for non-adherence was the MFS, which was considered as "boring" and "strenuous" in terms of having to answer multiple questions. By comparison, the cognitive tests were perceived to be "fun" and "enjoyable". The questionnaire also took the longest out of all four tasks to complete. The shortcomings of the application were predominantly focused on the questionnaire rather than its overall ease of use.

Overall daily adherence by participants was $24 \%$. Daily reminders were issued at 15:00 and resulted in a participant adherence rate of $23 \%$ within the first two hours of receiving the reminder; this indicates that a large proportion of application use was in direct response to the daily reminders.

Analysis of the results obtained from the study indicates that the PVT, which is measured to $1 / 100$ second, has the strongest correlation with the MFS, measuring 0.293 with a significance of 0.008 . Figure 4 shows this positive liner correlation in data. This correlation may indicate that from a usability perspective the MFS could be replaced by the PVT and still produce accurate fatigue analysis in a more user friendly manner. This concurs with the results from previous studies into reaction times, showing that PVT is accurate at determining the occurrence of mental fatigue. By comparison, the Spatial Span Task had a correlation to the MFS of -0.141 , with significance of 0.209, and the Serial Addition/Subtraction Task was found to have a correlation to the MFS of -0.016 , with significance of 0.884 . Subsequently, both the Spatial Span Task and Serial Addition-Subtraction Task do not provide a strong correlation 
with the MFS. However, the correlation analysis would indicate PVT's potential to assess mental fatigue using a mobile device, as it is able to determine the current level of mental fatigue. A higher correlation of 0.342 for the MFS and PVT is observed when outliers were removed on the basis of inaccurate subjective-assessment, e.g. assessment scores were abnormally high or low when compared to testing scores, in conjunction with a simplistic pattern of responses observed (i.e. all responses were the lowest or highest possible choices). When further investigated, the majority of these outliers occurred by participants who reported that they arose in instances where the participants did not want to fill in the questionnaire and only wanted to take part in the other more engaging tasks. This would suggest that a questionnaire can be successfully adapted onto a mobile device, however, it doesn't guarantee that users will correctly engage with it, which could still potentially lead to a higher error rates in the scoring of results obtained from the questionnaire. On the other hand, the PVT showed promise as a potentially accurate and engaging way of assessing fatigue on a mobile device; participants were happier to engage with this task and it produced a correlative response to the validated MFS.

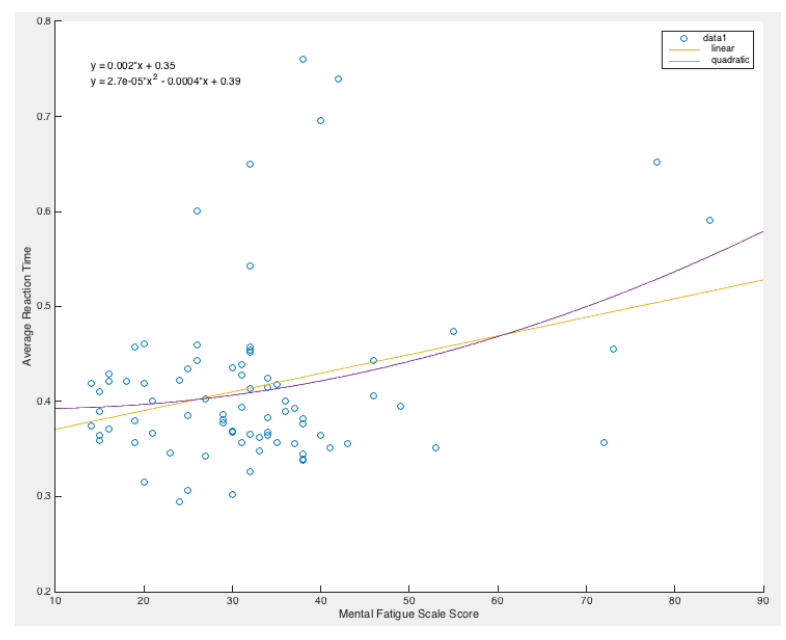

Fig. 4. MFS vs PVT Data Plot with Linear Correlation

\section{Conclusion \& Future Work}

The user experience design and efficacy of the proposed smartphone based cognitive fatigue objective assessment application have validated, through the use of an iterative multidisciplinary design methodology. However, a number of areas for improvement remain. User feedback indicated that carrying out the MFS was one of the least enjoyable parts of the overall process, which potentially reduces engagement in the process. As the use of PVT has been shown to provide similar assessment of mental fatigue, future work can exclude the use of MFS. Subsequently, this could further increase user participation rates, which, in turn, may potentially increase the accuracy of the 
mental fatigue evaluation. All three of the tasks employed resulted in positive user feedback, with some participants even indicating that they would like personal scores as it would further encourage them to participate more frequently. Consequently, by introducing a competitive aspect to the cognitive games, user effort and daily participation rates would potentially improve. Future work may additionally permit the availability of real-time data for relevant medical professionals, in order that effective and timely intervention could be arranged if any extreme fatigue becomes apparent.

The smartphone application presented in this research provides a potentially effective tool for the individual evaluation of fatigue levels in situations where formal intervention and assessment approaches are neither feasible, nor available. Furthermore, the smartphone application permits evaluation to be carried out on a continual daily basis. The study also proves that tradition self-assessment methods of fatigue assessment can be accurately replaced by more engaging test methods such as a PVT.

\section{References}

1. Lauren S. Aaronson, Cynthia S. Teel, Virginia Cassmeyer, Geri B. Neuberger, Leonie Pallikkathayil, and Anita Wingate Janet Pierce, Allan N. Press, Phoebe D. Williams. 1999. Defining and Measuring Fatigue. JOURNAL OF NURSING SCHOLARSHIP 31, 1: 45-50. http://doi.org/10.1016/j.apmr.2013.02.026

2. Johansson B. and Ronnback L. 2014. Mental fatigue scale and its relation to cognitive, social and emotional functioning after a TBI or stroke. Brain Injury 28, 1: 572-573. http://doi.org/10.4172/2329-9096.1000182

3. John Brooke. 1996. SUS-A quick and dirty usability scale. Usability evaluation in industry 189, 194: 4-7.

4. Yao-Jen Chang, Shu-Fang Chen, and Li-Der Chou. 2012. A feasibility study of enhancing independent task performance for people with cognitive impairments through use of a handheld location-based prompting system. IEEE transactions on information technology in biomedicine: a publication of the IEEE Engineering in Medicine and Biology Society 16, 6: 1157-63. http://doi.org/10.1109/TITB.2012.2198484

5. Barnan Das, Brian L. Thomas, Adriana M. Seelye, Diane J. Cook, Larry B. Holder, and Maureen Schmitter-Edgecombe. 2012. Context-aware prompting from your smart phone. 2012 IEEE Consumer Communications and Networking Conference (CCNC): 56-57. http://doi.org/10.1109/CCNC.2012.6181049

6. HPA Van Dongen, Greg Maislin, Janet M. Mullington, and David F. Dinges. 2003. The cumulative cost of additional wakefulness: dose-response effects on neurobehavioral functions and sleep physiology from chronic sleep restriction and total. Sleep 26, 2: 117126. Retrieved November 19, 2014 from http://www.med.upenn.edu/uep/user_documents/VanDongen_etal_Sleep_26_2_2003.pdf

7. Ann-Louice Lövgreen Engström, Jan Lexell, and Maria Larsson Lund. 2010. Difficulties in using everyday technology after acquired brain injury: a qualitative analysis. Scandinavian journal of occupational therapy 17, 3: 233-243. http://doi.org/10.3109/11038120903191806

8. Daniel Gartenberg and R McGarry. 2012. Development of a Neuroergonomic Application to Evaluate Arousal. Advances in ...: 6378-6387. Retrieved December 3, 2014 from http://books.google.com/books?hl=en\&lr=\&id=Plqeq0FmkIYC\&oi=fnd\&pg=PA239\&dq= 
Development + of $+a+$ Neuroergonomic + Application + to + Evaluate + Arousal\&ots $=\mathrm{s} 0 \mathrm{~N} 2 \mathrm{Ln} 1 \mathrm{U}$ jT\&sig=VCNgcFs-Wq4W3pf8kfpZTR-gJQg

9. Birgitta Johansson, Peter Berglund, and Lars Rönnbäck. 2009. Mental fatigue and impaired information processing after mild and moderate traumatic brain injury. Brain injury: [BI] 23, 13-14: 1027-40. http://doi.org/10.3109/02699050903421099

10. Birgitta Johansson, Helena Bjuhr, and Lars Rönnbäck. 2013. Evaluation of an Advanced Mindfulness Program Following a Mindfulness-Based Stress Reduction Program for Participants Suffering from Mental Fatigue After Acquired Brain Injury. Mindfulness. http://doi.org/10.1007/s12671-013-0249-z

11. Birgitta Johansson and Lars Rönnbäck. 2009. Mental Fatigue; A Common Long Term Consequence After a Brain Injury.

12. Birgitta Johansson and Lars Rönnbäck. 2012. Mental Fatigue and Cognitive Impairment after an Almost Neurological Recovered Stroke. ISRN Psychiatry 2012: 1-7. http://doi.org/10.5402/2012/686425

13. Birgitta Johansson, Anders Starmark, Peter Berglund, Martin Rödholm, and Lars Rönnbäck. 2009. A self-assessment questionnaire for mental fatigue and related symptoms after neurological disorders and injuries. Retrieved May 12, 2015 from http://informahealthcare.com/doi/abs/10.3109/02699050903452961

14. Matthew Kay and K Rector. 2013. PVT-touch: adapting a reaction time test for touchscreen devices. Pervasive ...: 248-251. Retrieved December 3, 2014 from http://ieeexplore.ieee.org/xpls/abs_all.jsp?arnumber=6563934

15. Krupp LB, LaRocca NG, Muir-Nash J, and Steinberg AD. The fatigue severity scale: Application to patients with multiple sclerosis and systemic lupus erythematosus. Archives of Neurology 46, 10.

16. K a Lee, G Hicks, and G Nino-Murcia. 1991. Validity and reliability of a scale to assess fatigue. Psychiatry research 36, 3: 291-298. http://doi.org/10.1016/0165-1781(91)90027$\mathrm{M}$

17. Ralph M Reitan and Deborah Wolfson. 1985. The Halstead-Reitan neuropsychological test battery: Theory and clinical interpretation. Reitan Neuropsychology.

18. Azmeh Shahid, Kate Wilkinson, Shai Marcu, and ColinM. Shapiro. 2012. Visual Analogue Scale to Evaluate Fatigue Severity (VAS-F). In STOP, THAT and One Hundred Other Sleep Scales SE - 100, Azmeh Shahid, Kate Wilkinson, Shai Marcu and Colin M Shapiro (eds.). Springer New York, 399-402. http://doi.org/10.1007/978-1-4419-9893-4_100

19. Ida Sue Baron. 2004. Delis-Kaplan Executive Function System. Child Neuropsychology 10, February: 147-152. http://doi.org/10.1080/09297040490911140

20. Dallas Swendeman, W. Scott Comulada, Nithya Ramanathan, Maya Lazar, and Deborah Estrin. 2014. Reliability and Validity of Daily Self-Monitoring by Smartphone Application for Health-Related Quality-of-Life, Antiretroviral Adherence, Substance Use, and Sexual Behaviors Among People Living with HIV. AIDS and Behavior 19, 2: 330-340. http://doi.org/10.1007/s10461-014-0923-8

21. David Wechsler. 2008. Wechsler adult intelligence scale-Fourth Edition (WAIS-IV). San Antonio, TX: NCS Pearson.

22. Carlo Ziino and Jennie Ponsford. 2005. Measurement and prediction of subjective fatigue following traumatic brain injury. Journal of the International ...: 416-425. Retrieved November 12, 2014 from http://journals.cambridge.org/abstract_S1355617705050472

23. ResearchKit Reference. Retrieved February 9, 2016 from http://researchkit.org/docs/index.html

iOS Human Interface Guidelines: Research Apps. Retrieved February 9, 2016 from http://researchkit.org/hig/index.html 